\title{
A violência doméstica em tempos de isolamento social: Uma revisão integrativa
}

\author{
Domestic violence in times of social isolation: An integrative review \\ Violencia doméstica en tiempos de aislamiento social: Una revisión integradora
}

Recebido: 26/02/2021 | Revisado: 04/03/2021 | Aceito: 11/03/2021 | Publicado: 18/03/2021

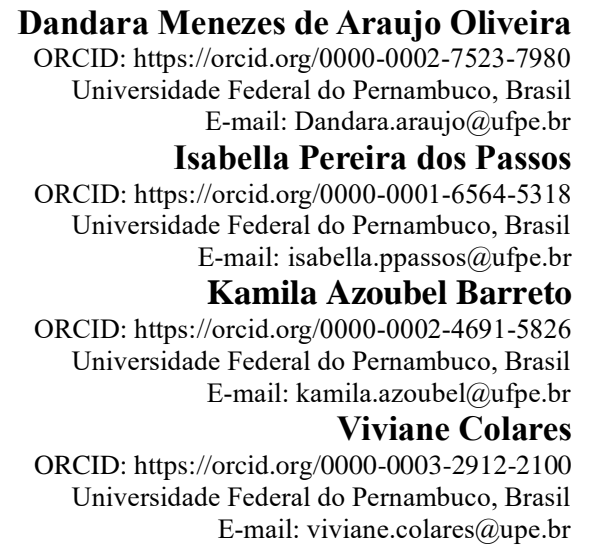

\begin{abstract}
Resumo
Objetivo: realizar uma compilação de dados acerca do isolamento social relacionado a pandemia do COVID-19 como um fator de risco para o aumento da violência doméstica sofrida por crianças e adolescentes. Metodologia: Foi realizada uma revisão integrativa da literatura através da busca ativa nas bases de dados BVS e Pubmed, além de consulta nas páginas online da OMS, UNESCO, UNICEF e Ministério da saúde. Como critérios de inclusão, foram selecionados artigos do ano de 2020, que relacionavam o isolamento social, COVID-19 e a violência doméstica contra crianças e adolescentes. Resultados: A pandemia do COVID-19 pode exacerbar ou desencadear diversas formas de violência, principalmente contra mulheres e crianças. São vários os motivos que levam a violência doméstica, entre eles estão: insegurança econômica e estresse relacionado à pobreza, quarentenas e isolamento social, disponibilidade reduzida de acesso aos serviços de saúde, além da dificuldade de assistência para os grupos de risco. Embora a violência doméstica tenha aumentado durante esse período de quarentena, as denúncias diminuíram, principalmente com relação às crianças e adolescentes, devido ao fechamento de escolas e a redução da rede de apoio formada por parentes e amigos da família. Conclusão: O isolamento social causado pela pandemia do COVID-19 se apresenta como um fator de risco para o aumento da violência doméstica contra crianças e adolescentes.
\end{abstract}

Palavras-chave: Adolescentes; COVID-19; Crianças; Isolamento social; Violência doméstica.

\begin{abstract}
Objective: to compile data on the social isolation related to the pandemic of COVID-19 as a risk factor for the increase in domestic violence suffered by children and adolescents. Methodology: An integrative literature review was carried out by actively searching the VHL and Pubmed databases, in addition to consulting the WHO, UNESCO, UNICEF and Ministry of Health online pages. As inclusion criteria, articles from the year 2020 were selected, which related social isolation, COVID-19 and domestic violence against children and adolescents. Results: The COVID-19 pandemic can exacerbate or trigger various forms of violence, especially against women and children. There are several reasons that lead to domestic violence, among them are: economic insecurity and stress related to poverty, quarantines and social isolation, reduced availability of access to health services, in addition to the difficulty of assistance for groups at risk. Although domestic violence has increased during this quarantine period, complaints have decreased, especially with regard to children and adolescents, due to the closure of schools and the reduction of the support network formed by relatives and friends of the family. Conclusion: The social isolation caused by the COVID-19 pandemic is a risk factor for the increase in domestic violence against children and adolescents.
\end{abstract}

Keywords: Adolescents; COVID-19; Children; Social isolation; Domestic violence.

\section{Resumen}

Objetivo: recopilar datos sobre el aislamiento social relacionado con la pandemia de COVID-19 como factor de riesgo para el aumento de la violencia intrafamiliar sufrida por niños, niñas y adolescentes. Metodología: Se realizó una revisión integradora de la literatura mediante la búsqueda activa en las bases de datos de BVS y Pubmed, además de consultar las páginas en línea de OMS, UNESCO, UNICEF y Ministerio de Salud. Como criterios de inclusión se 
seleccionaron artículos del año 2020, que relacionaban aislamiento social, COVID-19 y violencia intrafamiliar contra niños, niñas y adolescentes. Resultados: La pandemia de COVID-19 puede exacerbar o desencadenar diversas formas de violencia, especialmente contra mujeres y niños. Son varias las razones que conducen a la violencia doméstica, entre ellas se encuentran: la inseguridad económica y el estrés relacionado con la pobreza, las cuarentenas y el aislamiento social, la menor disponibilidad de acceso a los servicios de salud, además de la dificultad de asistencia a los grupos en riesgo. Si bien la violencia intrafamiliar ha aumentado durante este período de cuarentena, las denuncias han disminuido, especialmente en lo que respecta a niños y adolescentes, debido al cierre de escuelas y la reducción de la red de apoyo formada por familiares y amigos de la familia. Conclusión: El aislamiento social provocado por la pandemia COVID19 es un factor de riesgo para el aumento de la violencia intrafamiliar contra niños, niñas y adolescentes.

Palabras clave: Adolescentes; COVID-19; Niños; Aislamiento social; La violencia doméstica.

\section{Introdução}

Diferentes formas de violência existem em todos os países e envolvem todos os segmentos da sociedade. No caso da violência doméstica, na maioria das vezes é perpetrada contra mulheres e crianças; e tem consequências devastadoras para os indivíduos que são abusados (OMS, 1998).

A violência doméstica é caracterizada por um comportamento abusivo que ocorre em uma relação familiar ou entre casais que convivem na mesma residência. Esse comportamento se constitui como um problema de saúde pública mundial, com graves consequências psicológicas e/ou físicas (Bott, Guedes, Ruiz-Celis, \& Mendoza, 2019).

As consequências da violência domésticas em crianças afetam tanto o seu presente como seu futuro. Segundo Storz (2020) os efeitos imediatos já são preocupantes e essa vivencia violenta na infância ainda trazem danos a longo prazo. Situações adversas vividas durante a infância causam distúrbios na saúde mental do adulto, podendo aumentar o risco de depressão, suicídio, obesidade ou uso de drogas.

Algumas situações, como o isolamento social ou quarentena devido à pandemia pelo COVID-19, tende a elevar os números estatísticos relacionados à violência doméstica, uma vez que as vítimas estão em constante convivência com o agressor, além de dificultar a denúncia e a assistência para os grupos de risco, como o fechamento das escolas e a diminuição da rede de apoio formada por parentes e amigos da família (Eyzaguirre, Rosales, \& Arroyo, 2020).

No Brasil em julho de 2020 foi sancionada a Lei n 14.022 que visa combater a violência doméstica e familiar contra mulheres, crianças, adolescentes, idosos e pessoas com deficiência durante a emergência de saúde pública de importância internacional decorrente do coronavírus. Essa lei propõe-se a tratar como urgente a prestação de serviços às vítimas, e enquanto durar o estado de emergência de saúde, todos os prazos processuais, apreciação de matérias, atendimento às vítimas e a concessão de medidas protetivas que tenham relação com atos de violência doméstica e famíliar.

As medidas de distanciamento e isolamento social para conter o contágio pela COVID-19, junto com a diminuição de recursos essências e as consequências econômicas dessas medidas, levam a vivencias de situações estressantes. Apesar do isolamento social ser uma medida compreensível para o controle da infecção; as consequências sociais, econômicas e psicológicas são intensas e podem ser um estímulo para violência doméstica (Usher, Bhullar, Durkin, Gyamfi \& Jackson, 2020).

O objetivo deste trabalho foi realizar uma compilação de dados acerca do isolamento social relacionado a pandemia do COVID-19 como um fator de risco para o aumento da violência doméstica sofrida por crianças e adolescentes.

\section{Metodologia}

Trata-se de um estudo de revisão da literatura, através do qual se reúnem as informações coletadas em artigos científicos, que vão fornecer o embasamento teórico para responder a pergunta condutora do estudo. Assim, o relato de revisões da literatura sobre determinado tema, é extenso, aprofundado e detalhado, refletindo os achados de diferentes pesquisadores (Pereira et al, 2018). 


\section{Estratégia de busca:}

A revisão de literatura foi realizada a partir da busca ativa de informações na Biblioteca Virtual de Saúde (BVS) e Pubmed. Para tanto, foram adotados os seguintes descritores (DeCs/MeSH): "criança", "adolescente", "violência doméstica", "isolamento social", "quarentena", "COVID-19" para a base de dados do BVS e "child", "adolescent", "minors", "quarantine", "social isolament" e "domestic violence", "COVID-19" na base de dados PUBMED; combinados pelos operadores booleanos AND e OR. Durante o processo de busca também foram incluídas referências indicadas como "artigos similares" pela PUBMED, assim como foram feitas buscas nas referências de artigos selecionados. O processo de busca e análise foi conduzido por três pesquisadores e a última consulta às publicações foi realizada em setembro de 2020.

Além das bases de dados supracitadas, também foram consultados páginas na internet da Organização mundial de saúde (OMS), Fundo das Nações Unidas para a Infância (UNICEF), Organização das nações unidas (UNESCO) e Ministério da Saúde do Brasil (MS).

\section{Critérios de elegibilidade dos artigos:}

Foram incluídos estudos originais que tenham avaliado a violência doméstica durante o período de isolamento social na pandemia mundial de 2020. Foram incluídas publicações de artigos científicos nos idiomas inglês, espanhol e português. Foram excluídos os artigos que abordam sobre violência não relacionado à situação de isolamento social decorrente da pandemia ou que não abordaram crianças e adolescentes.

\section{Resultados}

No processo de busca, foram identificadas 92 referências, e após análise foram selecionados 6 textos para elaboração deste trabalho (Figura 1) (Quadro 1). Além disso, foram utilizados dados de órgãos oficiais, como a Organização mundial de saúde (OMS), UNICEF, UNESCO (Quadro 2). 
Figura 1. Fluxograma de elegibilidade e inclusão dos artigos científicos.

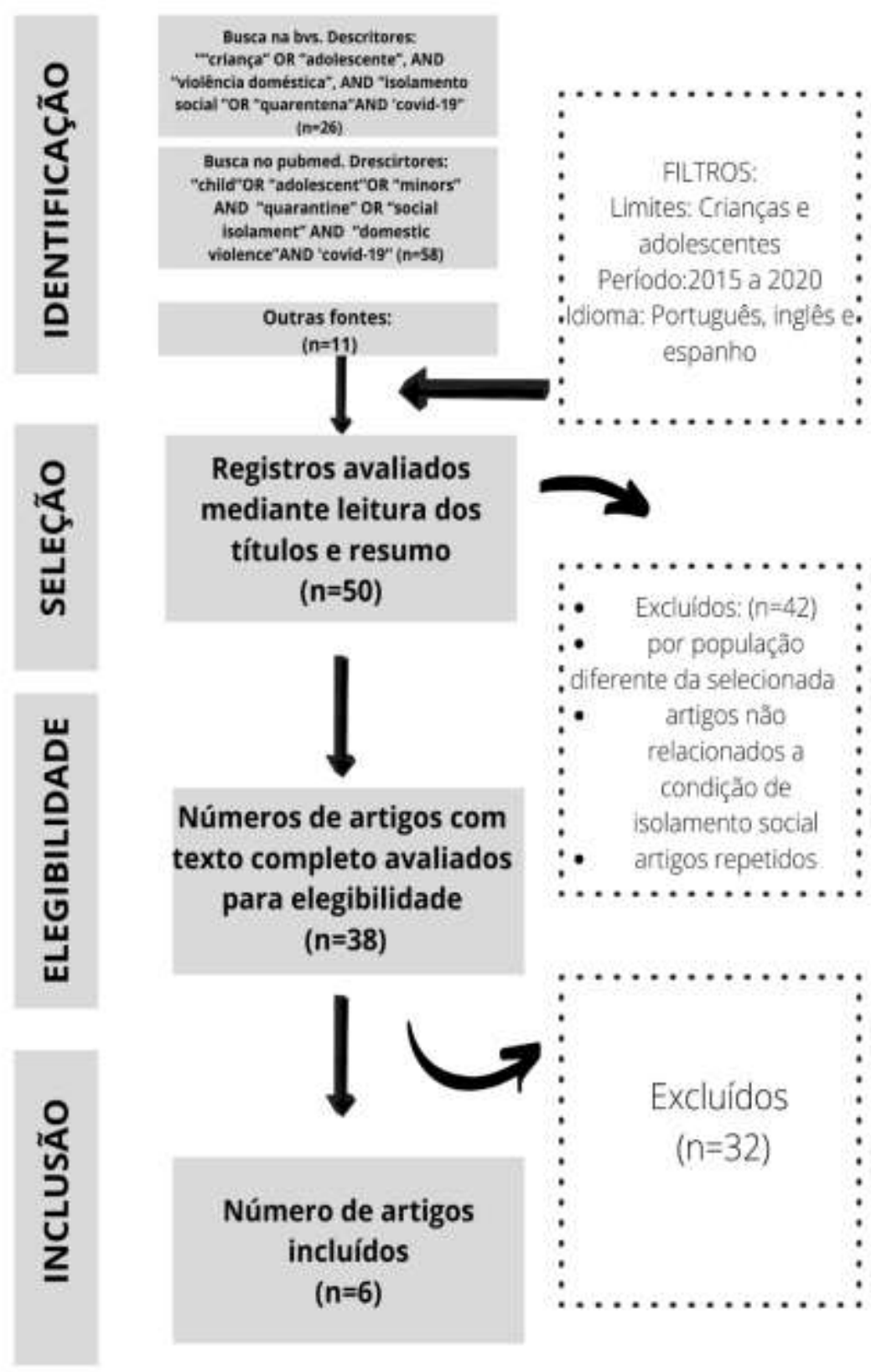

Fonte: Autores.

A Figura 1 exibe o fluxograma de elegibilidade e inclusão dos artigos científicos que foram utilizados na metodologia desse trabalho. 
Quadro 1. Distribuição dos estudos selecionados por autor, país, população-alvo e conclusão

\begin{tabular}{|c|c|c|c|}
\hline Autor(ano) & País & População-alvo & Conclusão \\
\hline Joanna Garstang et al., 2020 & GRÃ-BRETANHA & Crianças & $\begin{array}{l}\text { O fechamento da escola } \\
\text { devido ao COVID-19 pode } \\
\text { ter prejudicado as crianças, } \\
\text { pois o abuso infantil } \\
\text { permaneceu oculto. }\end{array}$ \\
\hline Acosta,Miguel, 2020. & ESPANHA & Mulheres & $\begin{array}{l}\text { O confinamento aumenta a } \\
\text { violência de gênero e seu } \\
\text { risco. }\end{array}$ \\
\hline Vieira et al, 2020. & BRASIL & Mulheres & $\begin{array}{l}\text { O Estado e a sociedade } \\
\text { devem ser mobilizados para } \\
\text { garantir às mulheres } \\
\text { brasileiras o direito a viver } \\
\text { sem violência. }\end{array}$ \\
\hline $\begin{array}{l}\text { Louis-Philippe Beland et al., } \\
2020\end{array}$ & CANADÁ & Famílias & $\begin{array}{l}\text { As preocupações das } \\
\text { mulheres em relação à } \\
\text { incapacidade de atender às } \\
\text { obrigações devido ao } \\
\text { COVID-19 leva a um } \\
\text { aumento significativo no } \\
\text { estresse familiar relatado e } \\
\text { violência doméstica. }\end{array}$ \\
\hline $\begin{array}{l}\text { Matteo Antonio Sacco et al., } \\
2020\end{array}$ & ITÁLIA & FAMÍLIA & $\begin{array}{l}\text { Os dados que mostram uma } \\
\text { diminuição no número de } \\
\text { denúncias de violência são } \\
\text { um alerta. É razoável } \\
\text { acreditar que as medidas de } \\
\text { restrição pessoal durante a } \\
\text { pandemia representam um } \\
\text { fator de risco perigoso para a } \\
\text { violência doméstica. }\end{array}$ \\
\hline Amber Peterman et al., 2020. & EUA & Mulheres e crianças & $\begin{array}{l}\text { Ainda não existem } \\
\text { avaliações rigorosas } \\
\text { específicas da pandemia } \\
\text { ligando essas } \\
\text { recomendações para } \\
\text { reduções causais em } \\
\text { violência contra mulheres e } \\
\text { crianças, incluindo detalhes } \\
\text { suficientes para orientar o } \\
\text { sequenciamento ou } \\
\text { cronograma de ações } \\
\text { durante o curso e pós- } \\
\text { pandemia. }\end{array}$ \\
\hline
\end{tabular}

Fonte: Autores.

O Quadro 1 exibe a distribuição dos estudos selecionados para esse trabalho, por autor, país, população-alvo e conclusão. 
Quadro 2. Distribuição das informações atribuídas aos órgãos oficiais.

\begin{tabular}{|l|l|l|l|}
\hline Orgão & País & População-alvo & Conclusão \\
\hline OMS & - & Crianças & $\begin{array}{l}1 \text { a cada 3 crianças sofrem de violência } \\
\text { devido a uma falha dos países em seguir } \\
\text { as estratégias para prevenir e proteger } \\
\text { esse grupo. }\end{array}$ \\
\hline EsSALUD & PERÚ & Mulheres e crianças & $\begin{array}{l}\text { O isolamento social aumentou a } \\
\text { vulnerabilidade e as vítimas de } \\
\text { violência doméstica. }\end{array}$ \\
\hline UNESCO & - & Crianças & $\begin{array}{l}\text { A pandemia do COVID-19, que levou } \\
\text { ao fechamento das escolas, limitou } \\
\text { ainda mais as fontes de apoio para esses } \\
\text { indivíduos, diminuindo a capacidade } \\
\text { dessas vítimas de lidar com a violência }\end{array}$ \\
\hline $\begin{array}{l}\text { Ministério da saúde } \\
\text { (MS) }\end{array}$ & BRASIL & Mulheres e crianças & $\begin{array}{l}\text { Aumento da violência doméstica e } \\
\text { diminuição dos serviços de apoio às } \\
\text { vítimas, principalmente nas áreas de } \\
\text { assistência social, saúde, segurança } \\
\text { pública e justiça }\end{array}$ \\
\hline
\end{tabular}

Fonte: Autores.

O Quadro 2 relata as informações coletadas para esse trabalho através das páginas eletrônicas dos órgãos oficiais, como OMS, EsSALUD, UNESCO e Ministério da Saúde.

\section{Discussão}

A violência doméstica pode ser definida como qualquer comportamento abusivo que ocorre com indivíduos habitando em uma mesma residência. Esse tipo de violência pode acontecer com qualquer indivíduo. No entanto, as crianças e adolescentes estão entre os grupos mais acometidos por esse mal (Sacco, Caputo, Ricci, Sicilia, Aloe, Bonetta, Cordasco, Scalise, Cacciatore, Zibetti, Gratteri \& Aquila, 2020). Existem várias maneiras pelas quais a violência doméstica pode ocorrer, são elas: física, sexual, psicológica e de perseguição (OMS, 1998).

A violência física pode ocorrer através de espancamentos, tortura e até assassinato. Enquanto que a violência sexual acontece através de relação sexual indesejada e/ou assédio (verbal ou físico), que quando acomete crianças, se caracteriza como pedofilia. A violência psicológica pode ocorrer através de manipulação, ameaças, humilhações e intimidações. Por último, a violência por perseguição se dá quando o agressor tem atitudes de perseguição e controle obsessivo de telefonemas ou mensagens (Sacco et al., 2020) .

Existem diversos comportamentos, desde os mais sutis aos mais expostos, que podem se caracterizar como violência doméstica. O comportamento abusivo tem uma espécie de "trajetória", onde se inicia por constrangimento da vítima, seguida de isolamento, menosprezo, até se consolidar com a prática abusiva. Ainda de acordo com esse autor, o cenário intrafamiliar revela uma dinâmica relacional de frustração crônica, estresse e decepção, podendo exacerbar o controle possessivo e destrutivo do agressor contra suas vítimas. Esse agressor pode ainda apresentar problemas psicológicos ou psiquiátricos. Nesse caso, há a possibilidade de situações como isolamento influenciarem negativamente seus comportamentos (Sacco et al., 2020). 
A situação da pandemia constitui um ambiente que pode exacerbar ou desencadear diversas formas de violência, inclusive violência contra mulheres e crianças. De acordo com Peterman, Potts, O’Donnell, Thompson, Shah, Oertelt-Prigione e Gelder (2020), algumas vias (diretas e indiretas) ligam a pandemia à violência contra mulheres e crianças, como insegurança econômica e estresse relacionado à pobreza, quarentenas e isolamento social, disponibilidade reduzida de serviços de saúde e acesso, incapacidade de mulheres para escapar temporariamente de parceiros abusivos, e violência perpetrada contra profissionais de saúde.

Num estudo realizado por Garstang, Debelle, Anand, Armstrong, Botcher, Chaplin, Hallett, Morgans, Price, Tan, Tudor e Taylor (2020) na Inglaterra foi detectado que durante lockdown da COVID-19 de março a junho de 2020 as recomendações de proteção a crianças diminuíram em comparação aos anos de 2018 e 2019, devido ao fechamento das escolas. Pois é nesse local que crianças e adolescentes encontram adultos confiáveis fora do seu ambiente familiar para relatar os abusos sofridos dentro de casa. A maioria das crianças sofriam algum tipo de violência, seja ela doméstica, física ou emocional, além de negligência.

Durante a pandemia da COVID-19 houve um aumento da violência de gênero sofrida por mulheres devido a impunidade do agressor e, o isolamento social constitui-se como uma barreira para que as mulheres saíssem do local da agressão e realizassem a denúncia. Além disso, esse tipo de violência traz um impacto negativo para crianças e adolescentes que vivem nesses lares, uma vez que estão expostos a um ambiente de medo, ameaças e até agressões diretas, levando a alterações psicológicas e físicas (Acosta, 2020; Brasil, 2020).

Para Vieira, Garcia e Maciel (2020) o aumento da violência doméstica teve um aumento significativo devido a coexistência com o agressor, incertezas políticas e econômicas geradas pela pandemia do novo coronavírus. Corroborando com isso, Beland, Brodeur, Haddad e Mikola (2020) relatou que a pandemia do coronavírus gerou a quarentena em massa, levando a incertezas quanto à manutenção da renda familiar, que por sua vez, criam uma atmosfera mais hostil dentro do lar.

O estresse familiar causado pelo home office foi algo notado no Canadá, onde mulheres perderam seus empregos e não puderam contribuir no orçamento familiar o que estava diretamente relacionado ao aumento da violência doméstica, pois acabam passando mais tempo com seus agressores, além disso a diminuição do contato com parentes e amigos diminuiu a rede de apoio dessas famílias, uma vez que esses são um importante agente social de controle da violência doméstica (Beland et al. 2020).

No Brasil, existiu o contraste de aumento da violência doméstica e diminuição dos serviços de apoio às vítimas, principalmente nas áreas de assistência social, saúde, segurança pública e justiça. De acordo com Vieira et al. (2020) a Ouvidoria Nacional dos Direitos Humanos (ONDH), do Ministério da Mulher, da Família e dos Direitos Humanos (MMFDH), no período de $1^{\circ}$ e 25 de março, mês da mulher, relataram um crescimento de $18 \%$ no número de denúncias registradas pelos serviços Disque 100 e Ligue 1808. Notou-se que saúde e delegacias são com frequência os locais procurados pelas vítimas para relatar a violência doméstica, mas durante a pandemia houve uma baixa demanda desses serviços, devido ao medo de contágio pelo vírus. Por isso, em julho de 2020 foi sancionada a lei $n^{\circ}$ 14.022/20 que determina a agilidade do atendimento às vítimas de violência doméstica, desde prazos processuais até a concessão das medidas protetivas cabíveis.

Segundo Sacco et al., (2020), é necessário que a vítima de violência doméstica reconheça que vive em um ambiente violento para conseguir escapar desse cenário. Porém, infelizmente, por medo das repercussões ou do impacto que a sua ação possa ter na família, opta por não procurar a ajuda necessária, que se tratando de crianças e adolescentes ocorre principalmente no ambiente escolar (Peterman et al., 2020).

A pandemia do COVID-19, que levou ao fechamento das escolas, limitou ainda mais as fontes de apoio para esses indivíduos, diminuindo a capacidade dessas vítimas de lidar com a violência. Apesar disso, de acordo com esse órgão, houve um aumento significativo nas ligações para linha de apoio as vítimas de abuso infantil e violência contra parceiros íntimos (UNESCO, 2020). 
Segundo a OMS (2020), aproximadamente 1 bilhão de crianças a cada ano sofrem de algum tipo de violência, sendo ela física, sexual ou psicológica, podendo levar a lesões, deficiências e até a morte. De acordo com os órgãos oficiais, OMS, UNICEF e UNESCO, esse fato se dá devido a uma falha dos países em seguir as estratégias para prevenir e proteger esse grupo.

Um relatório realizado por esses órgãos sinaliza uma necessidade clara, em todos os países, de intensificar os esforços para implementar essas estratégias. Apesar de a maioria dos países (88\%) terem leis importantes em vigor para proteger as crianças contra a violência, menos da metade (47\%) desses países estão aplicando-as com vigor (OMS, 2020).

A OMS e seus parceiros continuarão a trabalhar com os países para implementar totalmente as estratégias contra a violência infantil, melhorando a coordenação, desenvolvendo e implementando planos de ação nacionais, munindo a legislação específica com dados e resultados decorrentes de estudos técnicos. É necessária uma ação global para garantir que o apoio financeiro e técnico necessário uma ação global para garantir que o apoio financeiro e técnico necessário esteja disponível para todos os países. O monitoramento e a avaliação são cruciais para determinar até que ponto esses esforços de prevenção são efetivamente entregues a todos que deles precisam (OMS, 2020).

\section{Conclusão}

O isolamento social causado pela pandemia do COVID-19 se apresenta como um fator de risco para o aumento da violência doméstica contra crianças e adolescentes. Afastados do convívio com professores e colegas na escola e outros ambientes externos ao lar, ocorre a possibilidade do constante convívio com o ofensor; situação que pode ainda mascarar os números relacionados a esse tipo de violência, uma vez que dificulta a denúncia contra os agressores.

Diante do exposto, faz-se necessário novos estudos acerca do tema apresentado, de forma mais detalhada, para que novas estratégias possam ser delimitadas e, dessa forma, o problema em foco possa ser contido. Outras pesquisas devem ser conduzidas de forma que se possa melhor compreender o processo e os fatores relacionados à violência doméstica, assim como suas consequências sociais e sequelas no crescimento e desenvolvimento de crianças e adolescentes.

\section{Referências}

Acosta, M. L. (2020). Violencia de género en tiempos de pandemia y confinamiento. Revista Española de Medicina Legal.

Beland, L. P., Brodeur, A., Haddad, J., \& Mikola, D. (2020). Covid-19, family stress and domestic violence: Remote work, isolation and bargaining power.

Bott, S., Guedes, A., Ruiz-Celis, A. P., \& Mendoza, J. A. (2019). Intimate partner violence in the Americas: a systematic review and reanalysis of national prevalence estimates. Revista panamericana de salud publica, 43.

Brasil. (2020). Proteção: lei reforça medidas de combate à violência doméstica e familiar durante a pandemia.

Garstang, J., Debelle, G., Anand, I., Armstrong, J., Botcher, E., Chaplin, H., \& Taylor, J. (2020). Effect of COVID-19 lockdown on child protection medical assessments: a retrospective observational study in Birmingham, UK. BMJ open, 10(9), e042867.

Lei n 14.022, de 7 de julho de 2020. (2020) dispõe sobre medidas de enfrentamento à violência doméstica e familiar contra a mulher e de enfrentamento à violência contra crianças, adolescentes, pessoas idosas e pessoas com deficiência durante a emergência de saúde pública de importância internacional decorrente do coronavírus responsável pelo surto de 2019. Brasília, DF.

Matassini, S., Duffoó, M., \& Alvarez, V. (2020). Violencia de género/familiar en tiempos de cuarentena. Instituto De Evaluacion De Tecnologis En Salud E Investigacion, 1,14 .

Pereira A.S. et al. (2018). Metodologia da pesquisa científica. UFSM.

Peterman, A., Potts, A., O'Donnell, M., Thompson, K., Shah, N., Oertelt-Prigione, S., \& Van Gelder, N. (2020). Pandemics and violence against women and children (Vol. 528). Washington, DC: Center for Global Development.

Sacco, MA, Caputo, F., Ricci, P., Sicilia, F., De Aloe, L., Bonetta, C. F, \& Aquila, I. (2020). O impacto da pandemia Covid-19 na violência doméstica: O lado negro do isolamento doméstico durante a quarentena. Jornal Médico-Legal, 88 (2), 71-73.

Storz M. A. (2020). Child abuse: a hidden crisis during covid-19 quarantine. Journal of paediatrics and child health, 56(6), 990-991.

United Nations Educational, Scientific and Cultural Organization(2020). Covid-19 educational disruption and response. 
Research, Society and Development, v. 10, n. 3, e34810313343, 2021

(CC BY 4.0) | ISSN 2525-3409 | DOI: http://dx.doi.org/10.33448/rsd-v10i3.13343

Usher, K., Bhullar, N., Durkin, J., Gyamfi, N., \& Jackson, D. (2020). Family violence and COVID-19: Increased vulnerability and reduced options for support. International journal of mental health nursing, 29(4), 549-552.

Vieira, P. R., Garcia, L. P., \& Maciel, E. L. N. (2020). The increase in domestic violence during the social isolation: what does it reveals?. Revista Brasileira de Epidemiologia, 23.

World Health Organization (1998). Domestic violence: A priority public health issue in the Western Pacific Region.

World Health Organization (2020). Joint Leaders' statement - violence against children: a hidden crisis of the COVID-19 pandemic. Geneva: World Health Organization. 